\title{
CONFERENCIA
}

\section{UM PSIQUIATRA COMO PRECURSOR DE FREUD}

\author{
PAULO LENTINO *
}

Freud, sem ter sido um psiquiatra pròpriamente dito, fêz muito mais pela Psiquiatria do que poderiam fazer os mais brilhantes psiquiatras, pois que sua doutrina contribuiu para que a Psiquiatria se desenvolvesse vertiginosamente, tanto no setor puramente especulativo, como no dinâmico-interpretativo, mas principalmente no setor terapêtico, onde sua influência se tornou decisiva. Poetas e filósofos foram, indiscutivelmente, precursores de Freud, e é êle próprio quem admite, em sua autobiografia, as extensas concordâncias da Psicanálise com as filosofias de Schopenhauer e Nietzche. No entanto, não há qualquer referência a psiquiatra que pudesse ser considerado como seu precursor. Nesta breve comunicação queremos, a bem da verdade histórica, adjudicar êsse direito inconteste ao grande psiquiatra suiço Wilhelm Griesinger. Vamos primeiramente traçar, de maneira superficial, o perfil dêsse grande médico, louvando-nos principalmente das informações contidas na "História da Psicologia Médica", de Gregory Zilbborg.

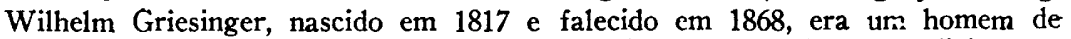
grande energia, de espírito independente e voluntarioso. Formado em medicina aos 21 anos, fêz um estágio na França e, depois, estabelecendo-se em Zurich, tornou-se professor da patologia interna, de clínica médica e de clínica psiquiátrica na Universidade de sua cidade natal. Dedicou-se especialmente ao estudo da fisiologia e editou o "Archiv für physiologische Heilkunde". Em 1845, com apenas 28 anos de idade, publicou notável livro - "Pathologie und Therapie der psychischen Krankheiten" - volume de 396 páginas. Em seguida, afastou-se das lides psiquiátricas, que só retomou em 1866; dado o renome que adquiriu, foi convidado a dirigir a divisão de enfermidades mentais no Charité de Berlim. Morreu dois anos depois, com a idade de 51 anos. Foi êle que introduziu o "nonrestraint" nos hospitais alemães; fundou a importante revista especializada "Archiv für Psychiatrie und Nervenkrankheiten"; desenvolveu grande labor em patologia cerebral; introduziu em Psiquiatria o conceito de predisposiçāo neuropática (apud Bumke): ao lado de Westphall, é considerado um dos fundadores da escola neurológica em Psiquiatria, sendo sua influência decisiva na Psiquiatria alemã.

Griesinger admitia que não conhecia psicologia, mas isso era modéstia de sua parte, pois que sua contribuição à Psiquiatria vem, muito ao contrário, demonstrar ser êle profundo conhecedor dos problemas psicológicos. Queremos demonstrar que Griesinger, além de ser um grande somatólogo, também era grande psicólogo, podendo ser considerado como o fundador da escola psicológica em Psiquiatria. Para tanto não vamos analisar tơda sua importante contribuição psicológica à Psiquiatria, mas apenas aquelas contribuições que o credenciam como precursor de

Trabalho apresentado no Centro de Estudos Franco da Rocha (Juqueri) em 25 setembro 1946. Entregue para publicação em 26 setembro 1946.

* Ex-psiquiatra do Hospital de Juqueri. 
Freud, transcrevendo os conceitos emitidos ou endossados por êle em seu substancioso tratado "Pathologie und Therapie der psychischen Krankheiten". na tradução francêsa de Doumic, da segunda edição alemã, anotada de maneira brilhante pelo grande Baillarger e publicada em Paris, em 1865. Passemos ao texto do citado livro, servindo-nos das próprias palavras do autor:

Já na página 30 , encontramos perfeita definição do Inconsciente, considerado sob um aspecto dinâmico e de sua importância em nossa vida mental: "Il y a dans l'intelligence une vie et un mouvement actifs, bien que nous n'en ayons pas conscience, mais que nous reconnaissons par leurs résultats. - Une activité constante règne dans cette sphère plongée dans les ténèbres ou le crépuscule, qui est beaucoup plus grande et plus caractéritique pour l'individualité que le nombre relativement petit d'idées passées à l'état de conscience. Une foule d'irritations physiques, d'impressions nées au sein même de l'organisme frappent tout d'abord et même pour ainsi dire exclusivement cette sphère, et agissent sur elle, sans que nous en ayons conscience, en modifiant les phénomènes, ies mouvements qui se produisent dans ce domaine, contribuent puissamment à déterminer la disposition actuelle du caractère; ce sont eux qui dirigent nos goûts, qui guident nos sympathies e nos antipathies."

A página 38, apresenta claramente uma idéia do que seja a repressão dos instintos: "Toutes les circonstances qui troublent la succession et l'enchainement normal des idées qui représentent le moi, et par conséquent qui entravent la liberté du moi, peuvent engendrer la douleur morale. Un excès d'irritation morale qui provoque de nouvelles idées arrivant confusément et sans ordre, comme aussi 1'absence de toute excitation, peut donner naissance à des sentiments pénibles (ennui, indifference), de même que dans le système nerveux sensitif, la douleur peut être le résultat aussi bien d'une irritation violente (impressions tumultueuses) que de l'absence des excitants ordinaires (le froid, la faim, etc.)."

A página 44, destaca a importância da "Fames et libido": "Quelques exemples (nautrages, etc.), ont montré que la faim portée à l'extrême repousse toutes les idées de morale ou de désintéressement qu'on lui oppose, et sans aller même jusqu'à ces actes de sauvagerie, c'est un fait vrai, bien que triste à dire pour des gens civilisés, que la faim et l'amour sont les motifs les plus puissants qui dirigent toutes nos actions."

A página 45, novamente nítida referência ao mecanismo básico da repressão, à dinâmica mental no sentido freudiano e, até, a utilização do termo complexo, bem mais tarde retomado por Jung: "Dans le désir et dans l'instinct, en tant qu'ils ne sont pas immédiatement satisfaits, certains complexus d'idées (relatives au but à atteindre) luttent contre les obstacles qui s'opposent à leur accomplissement́, et par là l'équilibre dans l'intensité relative des idées se trouve considérablement altéré. C'est pour cela que les instincts donnent très facilement naissancc à des émotions plus ou moins fortes, persistantes ou passagères (voy. $\S 30$ ), et, comc les instincts et les sentiments qu'ils éveillent viennent s'adjoindre à la perception, celle-ci en reçoit déjà un élément de mouvement qui tend à se manifester au dehors, la pensée a quelque chose de chaud, de sensuel, et ces mélanges donnent lieu à des états entièrement nouveaux de l'âme". Nesse final mostranos ter compreendido o fenômeno da "erotização do pensamento".

Sôbre a importância dos instintos e sôbre o mecanismo da catarse e da sublimação temos, na página 47 , prova inconteste da similitude de suas idéias com as de Freud: "Les idées se transforment en effort et en volonté par le fait d'une force interne dans laquelle nous reconnaissons encore pour la sphère intime de la vic de l'âme la loi fondamentale de l'action réflexe. Nous sommes forcés de vouloir. A l'état normal, cette force pousse l'homme à exprimir ses idées, à les réaliser par des actes et à les projeter ainsi au dehors. Cela fait, l'âme se sent soulagée, délivrée; elle s'est déchargée par ce fait de ses idées, et son équilibre 
est rétabli. C'est là un fait fondamental remarquable de la vie psychique et que chacun de nous doit connaitre pour l'avoir éprouvé. Ce fait se produit aussi bien chez l'artiste que son idéc, son désir de découvrir "un monde inconnu" ont occupé sans relâche pendant des années, mais que l'achèvement et la réussite de son oeuvre laissent froid et indifférent, que chez le malheureux dont l'esprit lutte longtemps contre la pensée d'un méfait, mais à qui l'accomplissement de cet act rend momentanéament le calme et la tranquillité".

$\mathrm{Na}$ página 49, ressalta a importância de um Ego forte, bem integrado: "Plus le moi est compact et homogène, plus le caractère est férme, plus aussi il apporte d'une manière tranchée son affirmation ou son veto dans la mise en oeuvre des idées qui se trouvent à l'état de fusion. Ainsi cette proposition: "la vraie liberté consiste dans la limitation", doit s'entendre particulièrement de cette convergence des pensées réalisée par he noi $i$; là où il n'y a pas de limitation, les idées surgissant constamment, souvent provoquées uniquement par les excitations actuelles des sens, ou traversant l'esprit d'une manière capricieuse, se pressent sans obstacle vers la face motrice de l'âme, et elles s'imposent. Les influences organiques les plus diverses peuvent diminuer, entraver ou mòme abolir complètement cette convergence des idées par le moi"'.

A página 55, emite idéias claras sôbre a diferenciação do Ego em função do meio, bem como sôbre os conflitos mentais e sua importância na dissociação psíquica: "Notre moi est, à différentes époques, très différent de lui-même; suivant l'âge, les divers devoirs de la vie, les évènements, les excitations du moment, tels ou tels complexus d'idées qui, dans un moment donné, représentent le moi, se développent plus que d'autres et se placent au premier rang. Nous sommes "un autre et cependant le même". Mon moi comme médecin, mon moi comme savant, mon moi sensuel, mon moi.moral, etc., c'est-à-dire les complexus d'idées, de penchants et de direction de la volonté, qui sont désignés par ces mots, peuvent entrer en opposition les-uns avec les autres et se repousser l'un l'autre à différents moments. Cette circonstance devrait avoir pour résultat non seulement l'inconséquence et la scission de la pensée et du vouloir, mais encore l'absence complète d'énergie sur chacune de ces faces isolées du moi, si, dans toutes ces sphères, il n'y avait pas un retour plus ou moins clair pour la conscience de quelques-unes de ces directions fondamentales".

A página 56, nos mostra como a ansiedade, a depressão pode resultar de um conflito íntimo que se manifesta na maior parte das psicoses: “...Ce n'est pas sans motif que nous avons choisi cet exemple très propre à expliquer la folie par des analogies nombreuses. Dans cette dernière, en effet, avec le début de la maladie cérébrale, il se développe ordinairement, également de l'intérieur vers l'extérieur des masses de sensations, de penchants et d'idées tout à fait nouveaux et qui, jusque-là, avaient été complètement inconnus sous cette forme à l'individu (par exemple sensations de grande anxieté auxquelles se lie l'idée d'un crime commis, l'idée de persécutions, etc.). Au début, ces idées nouvelles se présentent devant le moi ancien comme un toi étranger que souvent excite l'étonnement et la frayeur. Souvent les individus ressentent cette pénétration dans le cercle des idées anciennes, comme une prise de possession de l'ancien moi par une puissance obscure et irrésistible, et le fait de cette prise de possession est exprimé par des images fantastiques. Mais cette duplicité, cette lutte de l'ancien moi contre les nouveaux complexus d'idées non adéquates, s'accompagne toujours d'une sensation pénible de combat, d'état émotionnel et d'agitations violentes. C'est là en grande partie la cause de ce fait que l'expérience nous a appris, à savoir que les premières périodes de l'immense majorité des cas de folie consistent dans des affections des sentiments dont le caractére est ordinairement celui de la tristesse".

A página 60 , aponta-nos o perigo da não realização de um ideal, de um desejo: "Je connais un homme qui est devenu aliéné parce qu'il a été obligé de 
se faire boucher, alors qu'il voulait être ecclésiastique. - On trouve des faits semblables dans tous les asiles."

A. página 63, volta novamente ao mecanismo da repressão, do recalque: "Depuis la plus légère modification de la disposition de l'esprit jusqu'à l'émotion la plus furieuse, il n'y a donc que deux choses possibles: ou un état d'incitation et d'expansion du moi, dans lequel le moi se trouve bien, et par cela même se comporte affirmativement à l'égard du nouveau phénomène qui se passe dans la conscience et cherche à la retenir; ou bien un état d'empêchement, de répression, de dépression, dans lequel le complexus d'idées du moi, arrêté et refoulé dans son cours et dans sa transformation en efforts, s'envole aussitôt ou pénètre de force en luttant avec persévérance, et où le moi par conséquent se comport toujours d'une manière négative par rapport à ces nouvelles idées".

A. página 67, vemos, como também na citada pág. 63, que o têrmo e o conceito de recalque é seu: “...Mais quand l'ancien moi est vicié, corrompu, et faussé sous toutes ses faces par les idées fausses, morbides, quand en outre les complexus d'idées de l'ancien moi se trouvent si complètement refoulés (oubliés) que, sans la moindre trace d'émotion, le malade a échangé toute sa personnalité avec une autre et n'a plus guère que très-peu conscience de l'ancienne, la guérison est alors à peu près impossible, et ce n'est que très rarement que l'on arrive, en excitant chez eux de violentes émotions, et par une sorte de ỏressage mécanique (comme M. Leuret, par exemple, l'a tenté - Du traitement moral de la folic, Paris, 1840), à obtenir une rétrogradation notable des symptómes de la folie. $E_{t}$ même naturellement cela n'arrive que dans les cas où le cerveau n'a pas encore subi une lésion organique profonde; quand il existe une lésion de ce genre, comme dans la démence secondaire, il n'y a plus aucun espoir de guérison." ( $\mathrm{O}$ mecanismo da catarse também está aí esboçado).

A importância do princípio do prazer surge na página $\cdot 81$ : “Les conceptions délirantes favorables, gaies et heureuses, sont naturellement accueillies bien plus facilement et plus complètement dans le moi, qui se rend promptement à elles, après une courte résistance, et pałfois alors on voit l'esprit s'abandonner à ces conceptions délirantes, et se rlonger, avec une demi-conscience, dans un monde de rêves heureux."

A página 82 , nos dá uma indicação clara de como deveria ser realizada uma psicoterapia causal $\mathrm{c}$ individualista: "Presque toutes les idées fixes sont en dernièrc analyse l'expression d'un tort ou d'une satisfaction éprouvés dans les chose; qui intéressent les sentiments affectifs; aussi quand on les considère isolément, comme si elles étaient le fait capital dans la folic, arrive-t-on toujours à restreindre la question et à n'en voir qu'un seul côte, et $l^{\prime} \mathrm{cn}$ ne peut bien les comprendre et leur opposer un traitement médical convenable dans chaque cas en particulier, qu'en s'appuyant sur les états psychiques qui leur ont donné naissance".

O problema do sonho foi muito bem estudado e compreendido por Griesinger, coincidindo nos pontos básicos com as idéias de Freud, principalmente no que se refere à compreensão dos sonhos como realização de desejos insatisfeitos. Vamos transcrever apenas alguns tópicos das sete páginas que o autor dedica ao problema dos sonhos e de suas analogias com os estados psicopáticos: "Dans l'état de rêve, comme dans l'aliénation, on accepte tout, même les choses les plus bizarres et les plus singulières, comme des choses possibles, et l'on ne s'en étonne pas: 1a chose la plus absurde se transforme en une vérité indubitable quand la masse d'idées qui pourrait corriger cette erreur reste inactive". "Les rêves très agréables, ravissants, lumineux, sont très rares dans l'état de santé, le plus souvent ils surviennent à la suite d'un épuisement physique ou moral profond, et nous voyons souvent ici des idées qui étaient supprimées pendant la veille se reproduire d'une façon dominante en images de rêves. Au malhereux qui souffre physiquement et moralement, le rêve procure ce que la réalité lui refusait, le bien-être et le bonheur. Trenk en prison et souffrant de la faim rêvait souvent qu'il fasait de splendides repas; !e 
mendiant rêve qu'il est riche; celui à qui la mort a enlevê un être qu'il chêrissait, rêve volontiers qu'il lui est uni d'une manière intime et pour toujours, etc... De même chez les aliénés, l'état d'émotion douloureuse morbide auquel ils sont en proic, l'état de rêve profond dans lequel ils sont plongês, fait naitre des idées et des sentiments tout opposés, provoque des images lumineuses de bonheur, de grandeurs, d'honneurs, de richesse, etc., et dès que, par suite d'un changement survenu dans l'état du cerveau (mais sans qu'il y ait guérisson), j'oppression des sensations douloureuses disparait, la misère morale qui existait antêrieurement se transforme volontiers en une manifestation bruyante de l'orgueil et de la présomption des maniaques. C'est ainsi que l'on voit en particulier la possession supposée de richesses, l'accomplissement imaginaire de choses que l'on désirait, et dont la non-réalisation constituait un élément moral de la folie, faire si souvent le sujet principal du délire des aliénés; c'est ainsi que l'on voit une femme qui a perdu son enfant délirer dans le sens de la joie maternelle, un homme qui a perdu sa fortune se croire extraordinairement riche, la fille trompée se croire tendrement aimée par un amant fidèle, etc." (pág. 128). "Une foule d'autres phénumènes du rêve et la folie présentent encore une grande analogie. Ainsi quelquefois les alienés, comme les individus qui rêvent. n'ont aucune idée des mesures de temps, les minutes deviennent des heures. de même qu'en rêve nous traversons des années entières en un quart d'heure, et des évènements qui, pour leur accomplissement réel, demanderaient des mois, semblent pour l'aliéné s'être passés dans un délai très court. Dans ces deux états, les sensations musculaires (qui font croire que l'on vole, que l'on se précipite, etc.) et les hallucinations des sens jouent le rôle principal, et ces dernières servent en particulier à exprimer certaines situations qui sont provoquées par une disposition dominante de l'esprit comme correspondantes à celle-ci, tandis que les complexus d'idées du moi, qui pourraient mettre de l'ordre dans ce chaos, sont affaiblis ou détruits, luttent d'une façon douloureuse avec le nouveau fond de la vie de l'âme, ou sont violemment entrainés par ces nouvelles idées dans une direction déterminée" (pág. 129).

Demonstrou que a histeria também incidia em homens: "L'hystérie s'observe quelquefois, mais beaucoup plus rarement, chez de jeunes hommes; $j$ 'en ai vu plusieurs exemples, notamment il y a peu de temps chez un jeune homme très ané-. mique, dont la femme était enceinte; il avait la sensation très nette de la boule hystérique; il avait aussi de fortes convulsions. Toutefo:s je ne sache pas que 'ion ait encore signalé des cas de folie hystérique chez l'homme."

Mesmo o sentido dos delírios não escapou à argúcia de Griesinger. E assim quc: se exprime em relação aos delírios dos melancólicos, à página 269: “Quant à la manière dont se produit ce délire, nous l'avons déjà signalée plusieurs fois. Le malade se sent en proie à la tristesse; or il est habitué à n'être triste que sous l'influence de causes fâcheusse; de plus, la loi de causalité exige que cette tristesse ait un motif, une cause, et avant qu'il s'interroge, à ce suget, la réponse lui arrive déjà: ce sont toutes sortes de pensées lugubres, de sombres pressentiments, des appréhensions, qu'il ccuve et qu'il creuse jusqu'à ce que quelques-unes de ces idées soient devenues assez fortes et assez persistantes pour se fixer au moins pendant quelque temps. Aussi ce délire a-t-il le caractère de tentatives que fait le malade pour s'expliquer son état".

Depois do que expusemos, parece não haver dúvida ter sido Griesinger um legítimo e credenciado precursor de Freud. Este pequeno trabalho não visa de forma alguma desmerecer o mérito de Freud: a obra por êle estabelecida está suficientemente consolidada para que possa ser abalada. No entanto, também é justo que se realce $o$ valor de Griesinger.

Uma interrogação que surge é a de saber se Freud conheceria ou não a contribuição de Griesinger, que viveu pouco antes dêle e publicou seu livro na mesma 
língua usada por Freud. No primeiro volume da Interpretação dos Sonhos, de Freud, página 102 da edição Ballesteros, há uma citação indireta de Griesinger, que vamos transcrever: "Este pasage de Radestock es la síntesis (pág. 111) que descubre con toda claridad la realización de deseos como un caracter de la representación, comun al sueño y a la psicosis. Mis propias investigaciones me han mostrado que en esta hipótesis puede hallarse la clave de una teoría psicológica del sueño y de la psicosis." Essa citação indireta de Griesinger através de um livro de Radestock, demonstra que Freud não conhecia diretamente o livro de Griesinger, pois que, do contrário, não haveria dúvida que se reportaria com freqüência a êsse autor, reconhecida a sua honestidade científica.

Resta saber-se se as idéias contidas no livro de Griesinger, são próprias ou endossadas. Tudo parece indicar que muitas daquelas idéias transcritas neste trabalhe, foram assimiladas por Griesinger nas obras de Schopenhauer, que êle cita com treqüência, principalmente no "Über den Willen in der Natur". O próprio Freud reconheceu, em sua autobiografia, ser Schopenhauer seu precursor.

Outras idéias contidas na obra de Griesinger são muito originais e devem ser próprias, particularmente aquelas do terreno psiquiátrico e não apenas psicológico. De qualquer maneira, entretanto, sejam aquelas idéias próprias de Griesinger, endossadas ou assimiladas por êle, seu mérito como precursor de Fisud não se modifica, pois que autor psiquiátrico algum contemporâneo de Griesinger emitiu conceitos tão profundos e tão precisos no terreno psicológico. A assimilação das idéias de Schopenhauer e o seu aproveitamento imediato na Psiquiatria, mostram bem a agilidade do espírito de Griesinger.

Para finalizar, julgamos que, se Freud ainda vivesse e comparasse as idéias emitidas por Griesinger com sua doutrina psicanalítica, não teria dúvida em reconhecê-lo como seu mais legítimo precursor, como o fêz com Schopenhauer e Nietzsche.

Rua Ministro Ferreira Alves, 397 - S. Paulo 